\title{
Pro-angiogenic activity of astragaloside IV in HUVECs in vitro and zebrafish in vivo
}

\author{
YI ZHANG $^{1 *}$, GUANG HU ${ }^{1 *}$, SHANG LI $^{1}$, ZHEN HUA LI $^{1}$, CHE OI LAM $^{1}$, SI-JIA HONG ${ }^{1}$, YIU-WA KWAN ${ }^{2}$, \\ SHUN WAN CHAN ${ }^{3}$, GEORGE PAK-HENG LEUNG ${ }^{4}$ and SIMON MING-YUEN LEE ${ }^{1}$ \\ ${ }^{1}$ State Key Laboratory of Quality Research in Chinese Medicine, Institute of Chinese Medical Sciences, \\ University of Macau, Taipa, Macau SAR; ${ }^{2}$ School of Biomedical Sciences, Faculty of Medicine, \\ The Chinese University of Hong Kong, Hong Kong SAR; ${ }^{3}$ State Key Laboratory of Chinese Medicine and \\ Molecular Pharmacology, Department of Applied Biology and Chemical Technology, \\ The Hong Kong Polytechnic University, Hong Kong SAR; ${ }^{4}$ Department of Pharmacology and \\ Pharmacy, Faculty of Medicine, The University of Hong Kong, Hong Kong SAR, P.R. China
}

Received August 8, 2011; Accepted November 24, 2011

DOI: $10.3892 / \mathrm{mmr} .2011 .716$

\begin{abstract}
Astragaloside IV (AS-IV) is a natural product isolated from the Chinese medical herb, Radix Astragali, which has been reported to be a potential candidate for treating diseases associated with abnormal angiogenesis; however, the effect of AS-IV on angiogenesis and its underlying mechanisms are yet to be fully elucidated. In the present study, we investigated the angiogenic effect of AS-IV in vitro using human umbilical vein endothelial cells (HUVECs), and in vivo using zebrafish. AS-IV was found to stimulate the proliferation and migration of HUVECs in an XTT assay and a wound healing migration assay, respectively. Moreover, AS-IV stimulated the invasive ability of HUVECs and significantly increased the mean tube length of HUVECs in Matrigel. AS-IV induced an angiogenic response in HUVECs and enhanced mRNA expression of
\end{abstract}

Correspondence to: Dr Simon Ming-Yuen Lee, State Key Laboratory of Quality Research in Chinese Medicine, Institute of Chinese Medical Sciences, University of Macau, Av. Padre Tomás Pereira S.J., Taipa, Macao SAR, P.R. China

E-mail: simonlee@umac.mo

*Contributed equally

Abbreviations: AS-IV, astragaloside IV; HUVEC, human umbilical vein endothelial cell; eNOS, endothelial nitric oxide synthase; Flt-1, fms-like tyrosine kinase; KDR, kinase-domain region; Flk-1, fetal liver kinase-1 in mice; NO, nitric oxide; PI3K, phosphatidylinositol-3-kinase; VEGF, vascular endothelial growth factor; VEGFR, vascular endothelial growth factor receptor; VRI, vascular endothelial growth factor receptor inhibitor; ISV, intersegmental vessel; SIV, subintestinal vessel; DLAV, dorsal longitudinal anastomotic vessel

Key words: astragaloside IV, angiogenesis, Radix Astragali, human umbilical vein endothelial cell, zebrafish vascular endothelial growth factor (VEGF) and a VEGF receptor known as kinase-domain region/fetal liver kinase-1/ VEGF receptor 2 (KDR/Flk-1/VEGFR2), as well as activation of Akt as demonstrated by quantitative real-time PCR and Western blot analysis, respectively. The AS-IV-induced proliferation of HUVECs was capable of being suppressed by a KDR inhibitor (SU5416) and an Akt inhibitor (SH-6). AS-IV also rescued blood vessel loss in $T g$ (fli-1:EGFP) zebrafish. Altogether, our results suggest that AS-IV exerts potential pro-angiogenic effects in vitro and in vivo, and that its pro-angiogenic activity probably involves both VEGF- and Akt-dependent signaling pathways.

\section{Introduction}

Angiogenesis, the growth of new capillaries accomplished by sprouting, bridging or intussusceptive growth from existing vessels, plays a crucial role in the development of numerous diseases (e.g., various cancers, diabetic retinopathy, macular degeneration, psoriasis and rheumatoid arthritis) $(1,2)$. Vascular endothelial growth factors (VEGFs) are probably the most important inducers of angiogenesis owing to their potency, selectivity for endothelial cells and their ability to regulate proliferation and migration of endothelial cells (3). Therefore, overexpression of VEGF or its receptors promotes blood vessel formation, whereas inhibition of these molecules suppresses angiogenesis (4). In addition, other angiogenic cytokines and signaling pathways are also capable of influencing angiogenesis, for example the activation of Akt has been shown to stimulate the growth and migration of endothelial cells $(5,6)$.

For centuries, the Chinese herb Radix Astragali has been prescribed for the treatment of general weakness, chronic illnesses and cardiovascular disorders. Astragaloside IV (AS-IV) (3-O- $\beta$-D-xylopyranosyl-6- $O$ - $\beta$ D-glucopyranosylcycloastragenol) (Fig. 1A) is a major saponin constituent of the Chinese medical herb, which is capable of inhibiting vasoconstriction and protecting the brain against ischemic injury (7), as well as possessing endothelium-depen- 
dent vasorelaxant activity (8). Previously, we demonstrated that Radix Astragali extract exerts pro-angiogenic effects via the VEGF-kinase-domain region (KDR) and phosphatidylinositol-3-kinase-Akt-endothelial nitric oxide synthase (PI3K-Akt-eNOS) pathways in human umbilical vein endothelial cells (HUVECs) (9). The objective of this study was to investigate the angiogenic effect and mechanism of action of AS-IV in an attempt to identify the active compound(s) responsible for the pro-angiogenic activity of Radix Astragali extract. For the first time, AS-IV was found to promote angiogenesis in vitro and in vivo via activation of the VEGF and Akt signaling pathways.

\section{Materials and methods}

Chemicals and reagents. AS-IV was obtained from the National Institute for the Control of Pharmaceutical and Biological Products (China). Kaighn's modification of Ham's F12 medium and calcein AM were purchased from Invitrogen (Carlsbad, CA, USA). Endothelial cell growth supplement, dimethyl sulfoxide (DMSO), heparin and gelatin were supplied by Sigma-Aldrich (St. Louis, MO, USA). The growth factor-reduced Matrigel ${ }^{\mathrm{TM}}$ basement membrane matrix, VEGF, BioCoat $^{\mathrm{TM}}$ Matrigel 24-well plates and the BioCoat Matrigel invasion chamber were obtained from BD Biosciences (Franklin Lakes, NJ, USA). VEGF receptor (VEGFR) tyrosine kinase inhibitor II (VRI), tyrosine kinase inhibitor II, SH-6 and SU5416 were obtained from Merck KGaA (Germany). Anti-p-Akt antibody, anti-Akt antibody and goat anti-rabbit IgG horseradish peroxidase (HRP)-conjugated antibody were all purchased from Cell Signaling Technology (Beverly, MA, USA).

Maintenance of zebrafish (Danio rerio) and its embryos. The enhanced green fluorescent protein (EGFP) is selectively expressed in the nucleus of endothelial cells of $T g(f l i-1: E G F P)$ zebrafish embryos. Zebrafish adults and larvae were maintained as described in the Zebrafish Handbook (10) and all animal experiments were conducted in accordance with the ethical guidelines of the Institute of Chinese Medical Sciences, Macau.

Embryo collection and drug treatment. Zebrafish embryos were generated by natural pair-wise mating (3-12 months of age) and grown at $28.5^{\circ} \mathrm{C}$ in embyro water. The embryos were collected, dechorionated and distributed into a 12-well microplate at $28 \mathrm{~h}$ post fertilization (hpf) with at least 15 fish in each well, and treated with $100 \mathrm{ng} / \mathrm{ml}$ tyrosine kinase inhibitor II (VRI, one of the VEGF receptor inhibitors) for $3 \mathrm{~h}$. The VRI was washed out and replaced with $0.1 \%$ (v/v) DMSO embryo medium containing various concentrations of AS-IV and maintained at $28.5^{\circ} \mathrm{C}$ for $24 \mathrm{~h}$. The embryos maintained in $0.1 \%(\mathrm{v} / \mathrm{v})$ DMSO embryo medium throughout the course of the experiment served as the vehicle control.

Morphological observation of zebrafish. Zebrafish embryos were removed from the microplates following drug administration, and observed for viability and morphological changes under a fluorescence microscope (Olympus IX81 Motorized Inverted Microscope, Japan), equipped with a digital camera
(DP controller, Soft Imaging System, Olympus, Japan). Images were analyzed with Axiovision 4.2 and Adobe Photoshop 7.0 software.

Preparation of reagents and culture. A stock solution of AS-IV (100 mg/ml) was prepared in DMSO.

HUVEC culture. HUVECs (HU-V-E12; ATCC, USA) were cultured in Kaighn's modification of Ham's F12 medium with $2 \mathrm{mM}$ L-glutamine, $1.5 \mathrm{mg} / \mathrm{ml}$ sodium bicarbonate, $100 \mu \mathrm{g} / \mathrm{ml}$ heparin, $30 \mu \mathrm{g} / \mathrm{ml}$ endothelial cell growth supplement and $10 \%$ (v/v) fetal bovine serum (FBS) at $37^{\circ} \mathrm{C}$ in a humidified $5 \% \mathrm{CO}_{2}$ atmosphere. Tissue culture flasks were pre-coated with $0.1 \%$ $(\mathrm{w} / \mathrm{v})$ gelatin.

HUVEC proliferation as determined by the XTT assay. The HUVECs were trypsinized and seeded at $10^{4}$ cells/well in 96-well gelatin-coated plates. Following incubation at $37^{\circ} \mathrm{C}$ for $24 \mathrm{~h}$, the complete medium was removed and replaced with low-serum $[0.5 \%(\mathrm{w} / \mathrm{v}) \mathrm{FBS}]$ medium. Cells were incubated at $37^{\circ} \mathrm{C}$ for a further $24 \mathrm{~h}$ to achieve a quiescent state and then the low-serum medium was replaced with low-serum medium containing various concentrations of AS-IV. Cells receiving $0.1 \%$ DMSO alone served as the vehicle control. For the inhibition assays, HUVECs were treated with inhibitors $(0.3 \mu \mathrm{M}$ SU5416 and $1 \mu \mathrm{M} \mathrm{SH}-6)$ for $30 \mathrm{~min}$ prior to AS-IV treatment $(100 \mu \mathrm{g} / \mathrm{ml})$. In addition, cells cultured in $20 \mathrm{ng} / \mathrm{ml} \mathrm{VEGF}$ served as the positive control. After $48 \mathrm{~h}$, cell proliferation was assessed by the XTT assay for $4 \mathrm{~h}$. The spectrophotometric absorbance of each well was measured with a Multilabel counter (Perkin Elmer, Singapore). The absorbance of the formazan product was measured at $490 \mathrm{~nm}$, and the reference wavelength was $690 \mathrm{~nm}$. Cell viability data are expressed as a percentage of the control.

HUVEC proliferation assay by cell counting. HUVECs were seeded in gelatin-coated, 24-well tissue culture-treated plates at $5 \times 10^{4}$ cells/well. Cells were incubated in complete medium for $24 \mathrm{~h}$ at $37^{\circ} \mathrm{C}$, incubated with $100 \mu \mathrm{g} / \mathrm{ml} \mathrm{AS-IV} \mathrm{for} 30 \mathrm{~min}$, then washed and trypsinized. The cell suspension from each well was collected in a $1.5-\mathrm{ml}$ tube and centrifuged at $300 \mathrm{x} \mathrm{g}$ for $4 \mathrm{~min}$. The supernatant was discarded and the cell pellet was suspended in phosphate-buffered saline (PBS). The cell number was determined by counting with a hemocytometer.

HUVEC tube formation assay. The effects of AS-IV on HUVEC differentiation was examined by in vitro tube formation on Matrigel (11). Confluent HUVECs were harvested and diluted $\left(1 \times 10^{5}\right.$ cells) in $500 \mu \mathrm{l}$ of low-serum medium containing 10-100 $\mu \mathrm{g} / \mathrm{ml}$ AS-IV, seeded in $1: 1(\mathrm{v} / \mathrm{v})$ Matrigel-coated, 24-well plates in triplicate and incubated for $6 \mathrm{~h}$ at $37^{\circ} \mathrm{C}$. Cells receiving $0.1 \%$ DMSO alone served as the vehicle control, and cells cultured with $20 \mathrm{ng} / \mathrm{ml}$ VEGF served as the positive control. The network-like structures were examined under an inverted microscope (Carl Zeiss Axiovert 200, HK). Tube-like structures were defined as endothelial cord formations that were connected at both ends. The number of branching points in three random fields in each well was quantified using the Metamorph Imaging Series software (Japan). 
A

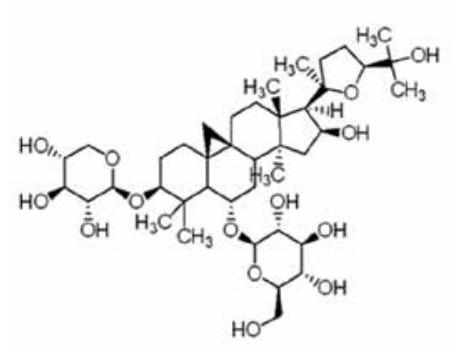

D
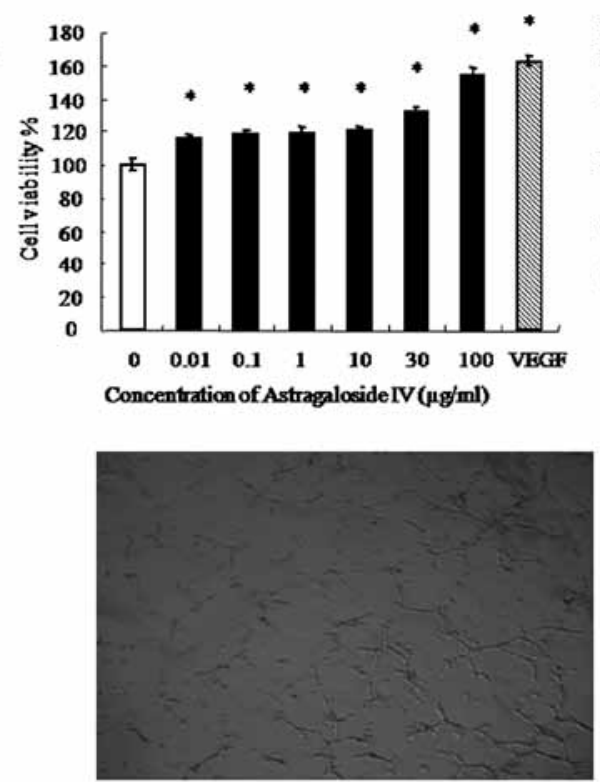

Control

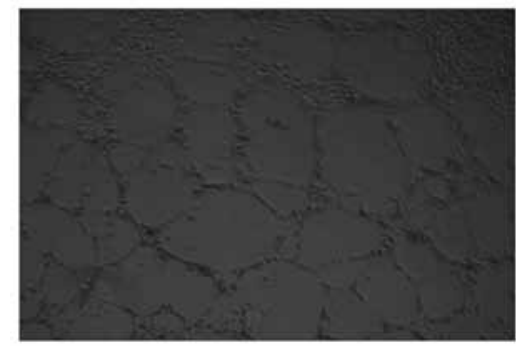

$10 \mu g / m l$ of AS-IV

B

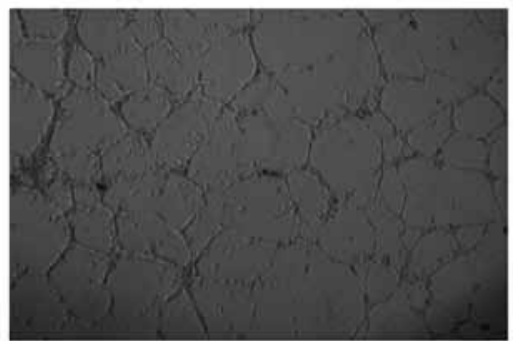

$30 \mu g$ minl of AS-IV
C
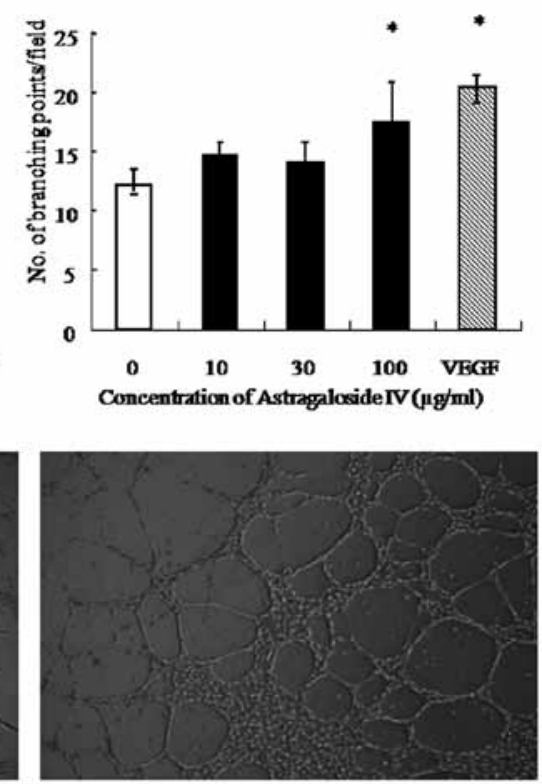

20ng/ml VECF

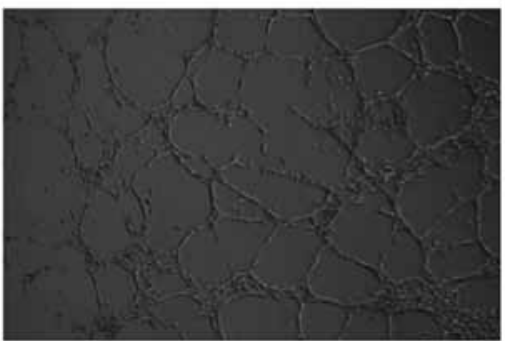

$100 \mu \mathrm{g} / \mathrm{ml}$ of AS-IV

Figure 1. AS-IV promotes HUVEC proliferation and tube formation. (A) Molecular structure of AS-IV. (B) Effect of AS-IV on the proliferation of HUVECs compared with the control group. (C) The number of branching points in HUVECs treated with various concentrations of AS-IV was calculated using Metamorph Imaging Series software. (D) Morphological features of AS-IV-treated HUVECs on Matrigel. Data are expressed as mean \pm SEM ( $n=3)$, $P<0.01$. VEGF, vascular endothelial growth factor; HUVECs, human umbilical vein endothelial cells; AS-IV, astragaloside IV.

HUVEC invasion assay. The HUVEC invasion assay was performed as described (9). The wells of the companion plate containing DMSO $(0.1 \%)$ served as the vehicle control and those containing $20 \mathrm{ng} / \mathrm{ml}$ VEGF served as the positive control.

HUVEC migration assay. Migration of HUVECs was examined by the wound healing method as described previously (12).

$m R N A$ expression analysis by real-time quantitative PCR. The mRNA expression analysis was conducted as described (9). The reaction without the complementary DNA (cDNA) product served as the negative control. The relative expression of VEGF and KDR was normalized to the amount of glyceraldehyde 3-phosphate dehydrogenase (GAPDH) in the same cDNA amount using the relative quantification method as described by the manufacturer.

Western blot analysis. HUVECs were treated with $100 \mu \mathrm{M}$ AS-IV for various lengths of time (10-60 min) and a medium containing $0.1 \%$ DMSO served as the control. The cells were then washed with PBS and lysed for 30 min on ice with lysis buffer [0.5 M NaCl, $50 \mathrm{mM}$ Tris, $1 \mathrm{mM}$ EDTA, $0.05 \%$ (w/v) SDS, $0.5 \%$ (v/v) Triton X-100, 1 mM PMSF, pH 7.4]. The cell lysates were centrifuged at $11,000 \mathrm{x}$ g for $20 \mathrm{~min}$ at $4^{\circ} \mathrm{C}$. Protein concentrations in the supernatants were measured using the bicinchoninic acid assay (Pierce, Rockford, IL, USA). The supernatants were subjected to sodium dodecyl sulfate polyacrylamide gel electrophoresis (SDS-PAGE) [12\% $(\mathrm{w} / \mathrm{v})$ polacrylamide gel], then transferred to polyvinylidene fluoride membranes, which were then blocked with $5 \%(\mathrm{v} / \mathrm{v})$ non-fat milk. Immunoblot analysis was commenced by incubation with anti-p-Akt and anti-Akt antibodies at $4^{\circ} \mathrm{C}$ overnight. Following washing, the membranes were incubated for $1 \mathrm{~h}$ at room temperature with HRP-conjugated goat anti-rabbit IgG. Proteins were detected using an advanced enhanced chemiluminescence (ECL) system (GE Healthcare, UK). Semiquantification was performed with densitometric analysis using Quantity One software (Bio-Rad, Philadelphia, PA, USA).

Statistical analysis. Each experiment was carried out in at least triplicate, and data are expressed as the mean \pm SEM of the control. The Student's t-test was used to analyze the data and the level of statistical significance was set at $\mathrm{P}<0.01$. 


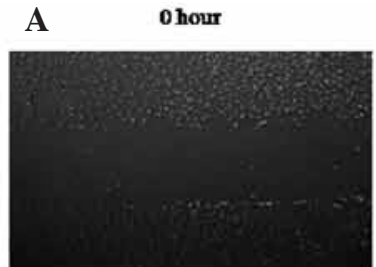

Control

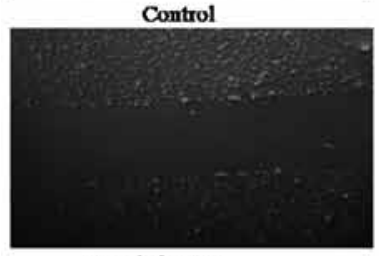

20ng/ml VEGF

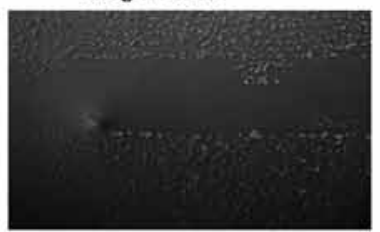

$100 \mu \mathrm{g} m \mathrm{ml}$ of AS-IV

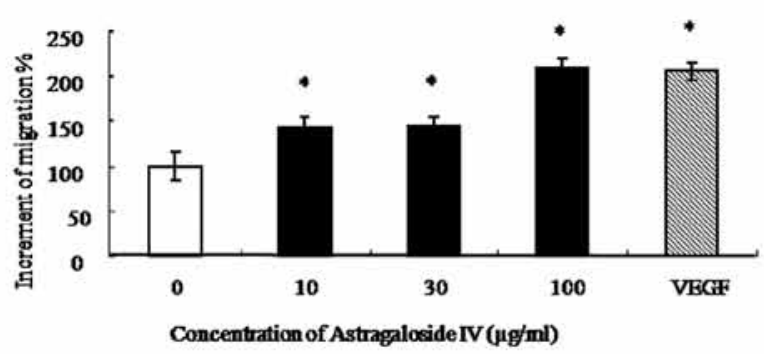

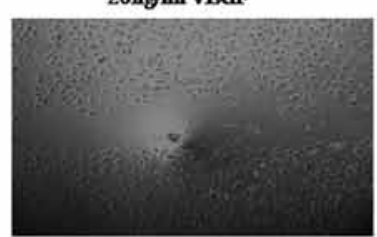

$100 \mu g$ ml of AS-IV
$\mathrm{C}$

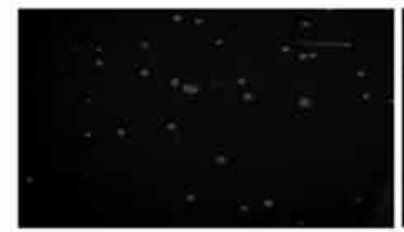

Control

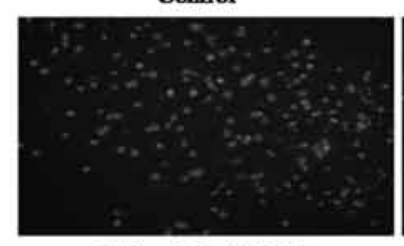

$100 \mu \mathrm{g} / \mathrm{ml}$ of AS-rV

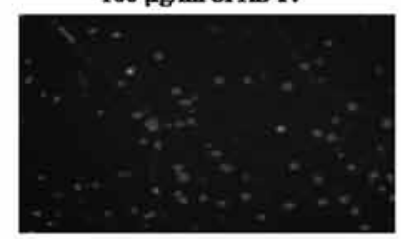

$10 \mu \mathrm{g} / \mathrm{ml}$ of AS-IV

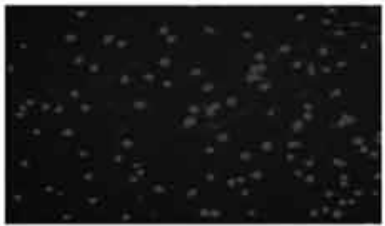

$20 \mathrm{ng} / \mathrm{ml}$ VEGF

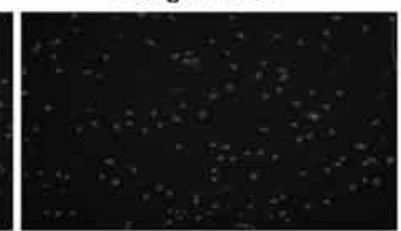

$30 \mu \mathrm{g} / \mathrm{ml}$ of AS-IV

D

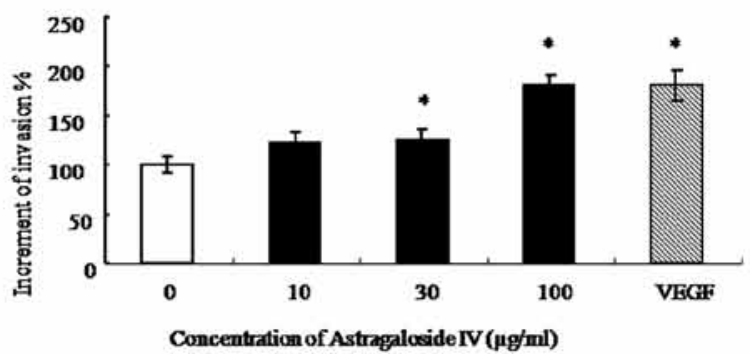

Figure 2. AS-IV enhances HUVEC migration and invasion. (A) Migration assay for AS-IV-treated endothelial cells. (B) The migratory ability was analyzed by averaging the mean length of the scraped area of each well compared to the control group. (C) Invasion assay for AS-IV-treated endothelial cells. (D) HUVEC invasion was quantified by counting the number of cells/insert using the Metamorph Imaging Series software. Data are provided as the mean \pm SEM ( $n=3),{ }^{*} \mathrm{P}<0.01$. VEGF, vascular endothelial growth factor; HUVECs, human umbilical vein endothelial cells; AS-IV, astragaloside IV.

\section{Results}

AS-IV promotes proliferation and tube formation in HUVECs. Metabolic rate, as an indirect indicator of cell number, was measured using the XTT assay. The amount of the formazan product in the cell culture medium revealed that proliferation of HUVECs was dose-dependent, with a 54\% increase in cell number at the high dose $(100 \mu \mathrm{g} / \mathrm{ml})$ of AS-IV (Fig. 1B).

The process of angiogenesis is complex and typically consists of proliferation and alignment to form tubular structures $(13,14)$. A Matrigel model was used in this study to determine the effect of AS-IV in inducing endothelial cell capillary tube formation. When HUVECs were cultured on Matrigel, which is a solid gel of mouse basement membrane proteins, they aligned easily and formed hollow, tube-like structures. As shown in Fig. 1C and D, there was little tube formation when HUVECs were plated on Matrigel in low-serum medium. Both the VEGF- and AS-IV-treated groups demonstrated marked morphogenetic changes in the tube formation of HUVECs. Fig. 1C and D shows that greater formation of branching points of the tube was found in the AS-IV-treated groups compared to the vehicle control. Statistical analysis of the quantitative measurements confirmed that AS-IV triggered a significant increase in terms of the number of branching points.

AS-IV enhances HUVEC migration and invasion in vitro. We determined the effects of AS-IV on endothelial cell migration using the wound-healing method. Fig. 2A illustrates that little HUVEC migration was measured in the vehicle control group at $16 \mathrm{~h}$ post-wounding, whereas a dramatic increment in HUVEC migration was measured in the treatment groups. Compared to the vehicle control group, AS-IV caused 44, 45 and $109 \%$ increases in HUVEC migration at concentrations of 10, 30 and $100 \mu \mathrm{g} / \mathrm{ml}$, respectively (Fig. 2B). Therefore, AS-IV significantly enhanced HUVEC migration in vitro.

We observed the effects of AS-IV on HUVEC invasion using Transwell culture inserts. Compared to the vehicle control group, VEGF-treated cells, positive control, exhibited an incease in invasive ability. Notably, in terms of cell invasion, HUVECs in the AS-IV-treated groups demonstrated a similar pattern. Specifically, there were 22, 25 and $81 \%$ increases in the AS-IV-treated groups at concentrations of 10, 30 and $100 \mu \mathrm{g} / \mathrm{ml}$, respectively (Fig. 2C and D). 
A

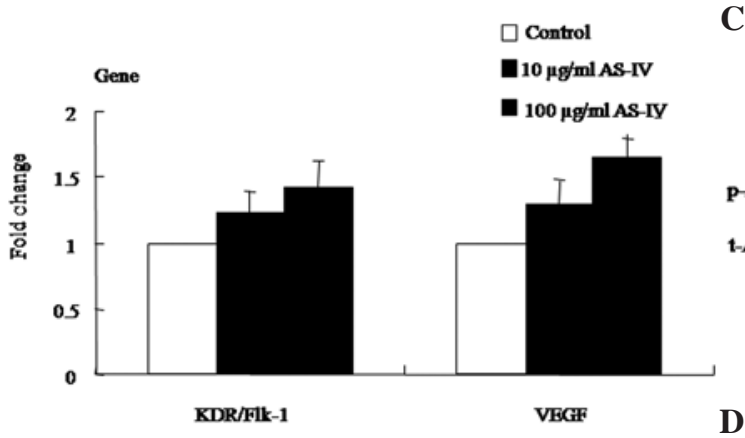

C

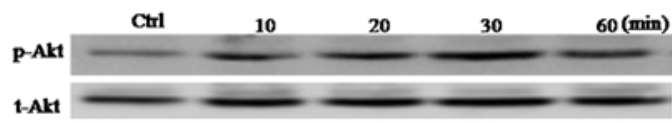

D
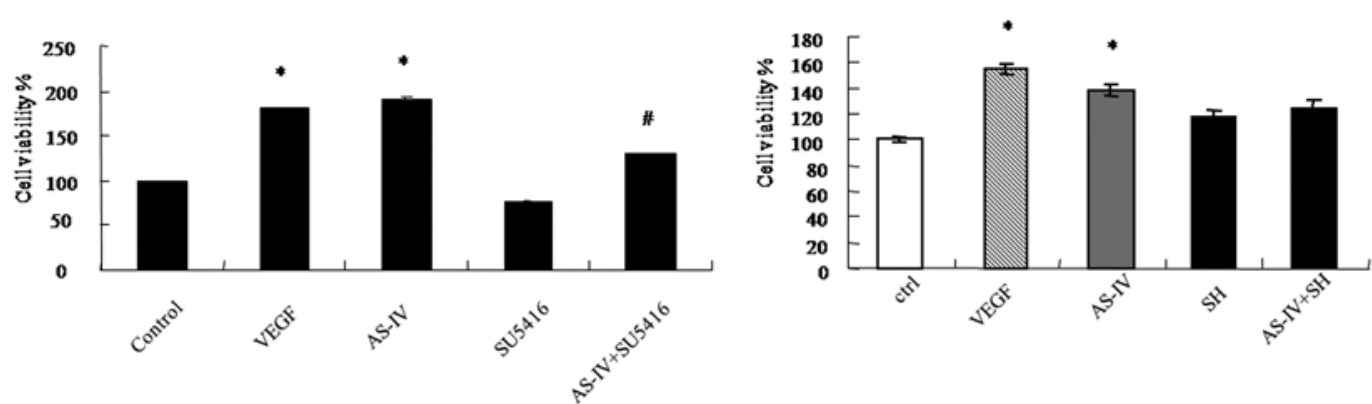

Figure 3. AS-IV exerts pro-angiogenic effects via VEGF- and Akt-dependent signaling pathways. (A) Gene expression in AS-IV-treated HUVECs. Data are provided as the mean \pm SEM from two individual experiments. (B) Effect of SU5416 on AS-IV-induced cell proliferation. HUVECs were pre-treated with SU5416 $(0.3 \mu \mathrm{M})$ for $30 \mathrm{~min}$ and then treated with AS-IV $(100 \mu \mathrm{g} / \mathrm{ml})$ for $48 \mathrm{~h}$. (C) Effects of AS-IV on Akt activation. (D) Effects of SH-6 on AS-IV-induced cell proliferation. Data are provided as the mean \pm SEM $(n=3),{ }^{*} \mathrm{P}<0.01$ versus control, ${ }^{\#} \mathrm{P}<0.05$ versus AS-IV. VEGF, vascular endothelial growth factor; HUVECs, human umbilical vein endothelial cells; AS-IV, astragaloside IV; KDR, kinase-domain region.

$A S$-IV increases VEGF and KDR $m R N A$ expression and VEGF receptor tyrosine kinase inhibitor abolishes AS-IV-induced HUVEC proliferation. We measured the mRNA expression of VEGF and KDR using real-time quantitative PCR in order to identify the molecular targets for the angiogenic effects of AS-IV on HUVECs. As shown in Fig. 3A, AS-IV up-regulated the gene expression of VEGF in a dose-dependent manner; VEGF expression was 1.2-fold and 1.7-fold higher than the control group at concentrations of 10 and $100 \mu \mathrm{g} / \mathrm{ml}$, respectively. KDR (also known as Flk-1 or VEGFR2), the major receptor responsible for the VEGF signaling pathway (15), also induced a dose-dependent increased gene expression in the AS-IV-treated groups.

To further examine whether the VEGF receptor plays a role in AS-IV-mediated angiogenesis, we investigated the effects of SU5416, a potent and selective inhibitor of the KDR receptor tyrosine kinase, on AS-IV-induced cell proliferation by the XTT assay. As shown in Fig. 3B, the proliferation of HUVECs was significantly increased $(\mathrm{P}<0.01)$ following treatment with AS-IV. Moreover, AS-IV-induced HUVEC proliferation was partially inhibited by SU5416 but no statistically significant difference $(\mathrm{P}<0.05)$ was noted between the SU5416+ASIV and ASIV groups. Thus, this evidence suggests that the pro-angiogenic activity of AS-IV may be mediated by KDR.

AS-IV stimulates HUVEC proliferation via Akt activation. The phosphorylation status of Akt, a central signaling molecule in regulating angiogenesis, in AS-IV-treated HUVECs was determined by Western blotting. As shown in Fig. 3C, AS-IV stimulated the phosphorylation of Akt in a time-dependent manner, which reached a plateau at $30 \mathrm{~min}$ and declined rapidly thereafter. The result also demonstrates that the total protein levels of Akt remained unaffected throughout the course of these experiments.

To further confirm whether AS-IV-mediated activation of Akt is essential to the angiogenic activity of AS-IV in HUVECs, we determined the effects of SH-6, a synthetic AKT inhibitor, on AS-IV-induced cell proliferation. SH-6 (1 $\mu \mathrm{M})$ partially reversed the proliferation of HUVECs induced by AS-IV (Fig. 3D). Overall, AS-IV promoted proliferation of HUVECs via activation of the Akt signaling pathway, in addition to stimulation of the KDR signaling pathway.

AS-IV rescues VRI-induced blood vessel loss in zebrafish. VRI, a pyridinyl-anthranilamide compound that displays anti-angiogenic properties, has been shown to potently inhibit the kinase activities of both VEGFRs 1 and 2 . The $28 \mathrm{hpf}$ zebrafish embryos, pre-treated with $300 \mathrm{nM}$ VRI for $3 \mathrm{~h}$, were placed into MilliQ-prepared water at $28.5^{\circ} \mathrm{C}$ for $24 \mathrm{~h}$ to allow development of blood vessel loss in regions of intersegmental vessels (ISVs) and dorsal longitudinal anastomotic vessels (DLAVs), as well as the formation of impaired subintestinal vessels (SIVs) (Fig. 4A). Following incubation the VRI-treated embryos with $30 \mu \mathrm{M}$ AS-IV instead of MilliQ-prepared water, the VRI-induced blood vessel loss at ISV and DLAV regions was significantly rescued, and the impaired SIV branching was partially restored (Fig. 4D). However, contrary to our previous reports that pro-angiogenic chemicals stimulate vessel spike formation in SIVs of healthy zebrafish $(16,17)$, AS-IV did not produce the pro-angiogenic effect under the same conditions (Fig. 4E). 


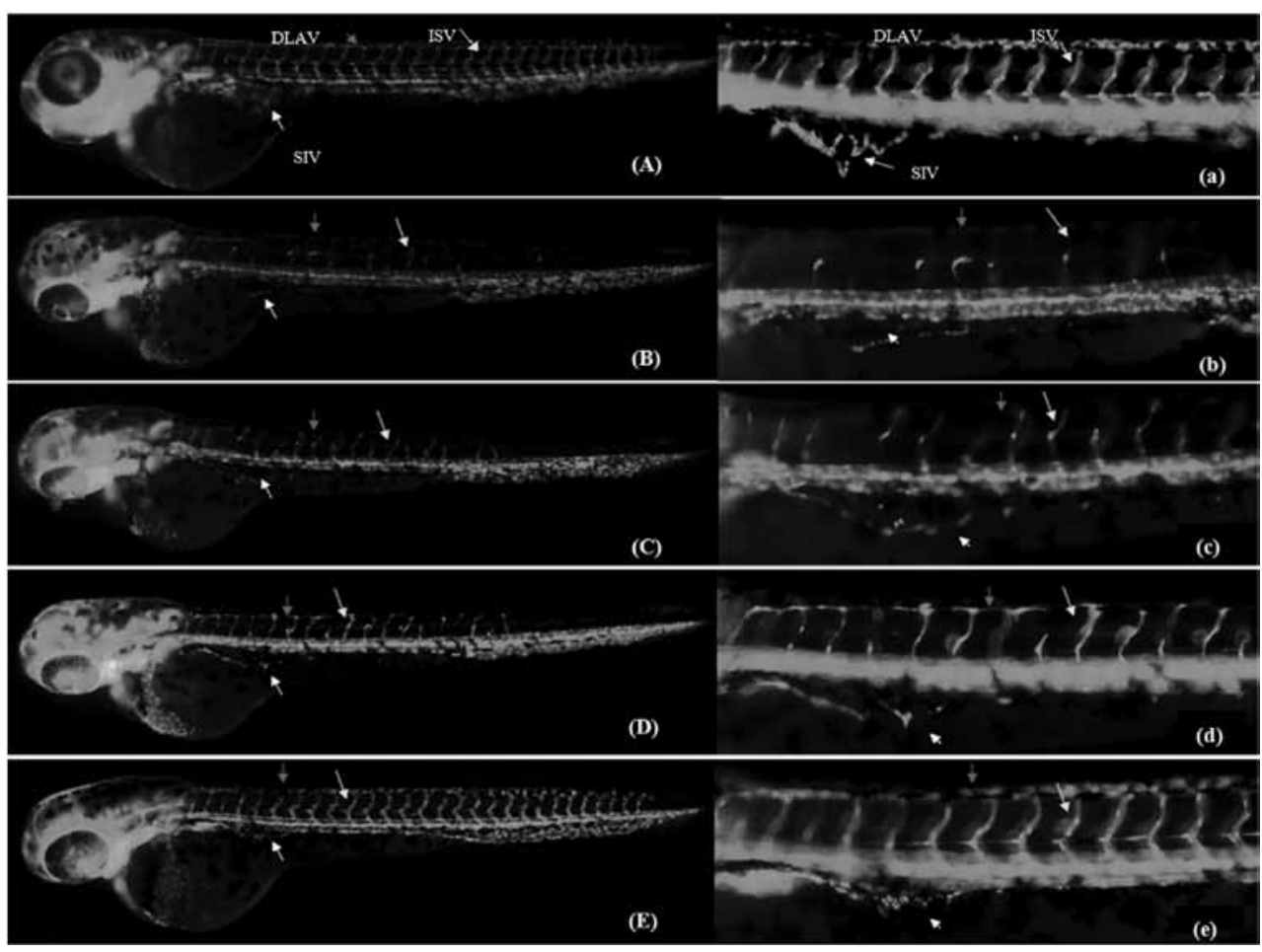

Figure 4. The pro-angiogenic effects of AS-IV on damaged blood vessels in $T g(f l-1: E G F P)$ zebrafish embryos. Zebrafish embryos (28 hpf) were treated with $300 \mathrm{nM}$ VRI for $3 \mathrm{~h}$ and then washed out. Pre-damaged blood vessel embryos were incubated with $0.1 \%$ (v/v) DMSO embryo medium, $10 \mu \mathrm{M}$ AS-IV or $30 \mu \mathrm{M}$ AS-IV for $24 \mathrm{~h}$. (A) Vehicle control: embryo (52 hpf); ISVs, SIVs and DLAVs appear as a well developed net structure. (B) VRI treatment only: treatment of embryo (28 hpf) with VRI for $3 \mathrm{~h}$ leads to reduced blood vessel formation in ISVs, SIVs and DLAVs. (C and D) Incubated VRI-treated embryo with 10 or $30 \mu \mathrm{M}$ AS-IV. Rescue of damaged blood vessels in ISVs and DLAVs by AS-IV is dose-dependent. (E) Healthy embryo (without VRI treatment) treated with $30 \mu \mathrm{M}$ AS-IV. Normal ISV, SIV and DLAV development was similar to that observed in the vehicle control. (a-e) Enlarged (x4.5) ISV region of A-E, respectively. White arrows, SIVs; red arrows, DLAVs; yellow arrows, ISVs. DMSO, dimethyl sulfoxide; AS-IV, astragaloside IV; VRI, VEGFR tyrosine kinase inhibitor II; hpf, h post fertilization; ISVs, intersegmental vessels; SIVs, subintestinal vessels; DLAVs, dorsal longitudinal anastomotic vessels.

\section{Discussion}

Radix Astragali is a potential angiogenic modulator that contains a considerable number of effective constituents (18). The angiogenic activity of the ethanol extract of Radix Astragali was further proved in multiple HUVEC angiogenesis assays in our previous study (9), and one active constituent, calycosin, was recently shown to be pro-angiogenic and to promote endothelial cell proliferation in both HUVECs and zebrafish larvae (19). In the present study, we identified another angiogenic compound, AS-IV, which acts differently in zebrafish in the angiogenesis assay in vivo compared with the effect of calycosin, since AS-IV promotes angiogenesis only in zebrafish that experienced vessel damage, rather than in healthy animals. Our results revealed that treatment with AS-IV promoted several features of angiogenesis in HUVECs in vitro and rescued VRI-induced blood vessel loss in transgenic zebrafish in vivo, and that this pro-angiogenic effect may involve activation of the KDR and Akt signaling pathways.

As demonstrated in Figs. 1 and 2, AS-IV-enhanced proliferation, migration, invasion and tube formation of HUVECs in vitro was dose-dependent. The results of real-time quantitative PCR illustrated that there were significant increases in mRNA expression of VEGF and its receptor (KDR) following treatment with AS-IV. In addition to the fact that AS-IV increased the expression of VEGF and KDR mRNAs, we found that the AS-IV-induced HUVEC proliferation was probably abrogated by SU5416, a selective inhibitor of KDR. All these pieces of evidence suggest involvement of the VEGF and KDR signaling pathways in AS-IV-induced angiogenesis.

Moreover, Fig. 3C and D demonstrates that Akt plays a significant role in AS-IV-induced angiogenesis. As well as the activation of KDR, VEGF has been reported to stimulate angiogenesis in endothelial cells via activation of Akt $(20,21)$. In the present study, the increased phosphorylation of Akt was triggered by AS-IV and the reversal of AS-IV-induced HUVEC proliferation by Akt inhibitors suggests that AS-IV activates angiogenesis via an Akt-related signaling pathway. Together, these results suggest that AS-IV is likely to induce angiogenesis involving activation of the VEGF-KDR and Akt signaling pathways.

Angiogenesis deficiency may result in numerous human vascular diseases (e.g., ischemic cardiac and cerebral problems). In the present study, VRI-induced blood vessel loss in zebrafish in vivo was capable of mimicking angiogenesis deficiency-associated human disease conditions. Our in vitro data clearly showed that AS-IV exhibited pro-angiogenic activity by stimulating HUVEC proliferation, migration and differentiation, whereas our in vivo data further suggest that AS-IV exerted a pro-angiogenic effect only in the damaged blood vessel disease model rather than in healthy zebrafish. This implies that AS-IV might be effective for the restoration of angiogenesis deficiency only under pathophysiological conditions, instead of in healthy subjects. In conclusion, these 
data suggest that AS-IV exerts its pro-angiogenic effects by rescuing damaged blood vessels. More investigations are required to elucidate the exact mechanism involved.

\section{Acknowledgements}

This study is supported by a grant from the Science and Technology Development Fund of Macau (ref. no. 045/2007/ A3) and Research Committee, University of Macau (ref. no. UL017).

\section{References}

1. Kahn J, Mehraban F, Ingle G, et al: Gene expression profiling in an in vitro model of angiogenesis. Am J Pathol 156: 1887-1900, 2000.

2. Milkiewicz M, Ispanovic E, Doyle JL and Haas TL: Regulators of angiogenesis and strategies for their therapeutic manipulation. Int J Biochem Cell Biol 38: 333-357, 2006.

3. Blázquez C, González-Feria L, Alvarez L, Haro A, Casanova ML and Guzmán M: Cannabinoids inhibit the vascular endothelial growth factor pathway in gliomas. Cancer Res 64: 5617-5623, 2004.

4. Ferrara N: Vascular endothelial growth factor: basic science and clinical progress. Endocr Rev 25: 581-611, 2004.

5. Dimmeler S, Fleming I, Fisslthaler B, Hermann C, Busse R and Zeiher AM: Activation of nitric oxide synthase in endothelial cells by Akt-dependent phosphorylation. Nature 399: 601-605, 1999.

6. Fulton D, Gratton JP, McCabe TJ, Fontana J, Fujio Y, Walsh K, Franke TF, Papapetropoulos A and Sessa WC: Regulation of endothelium-derived nitric oxide production by the protein kinase Akt. Nature 399: 597-601, 1999.

7. Luo Y, Qin Z, Hong Z, Zhang X, Ding D, Fu JH, Zhang WD and Chen J: Astragaloside IV protects against ischemic brain injury in a murine model of transient focal ischemia. Neurosci Lett 363: 218-223, 2004.

8. Zhang C, Wang XH, Zhong MF, Liu RH, Li HL, Zhang WD and Chen H: Mechanisms underlying vasorelaxant action of astragaloside IV in isolated rat aortic rings. Clin Exp Pharmacol Physiol 34: 387-392, 2007.

9. Zhang Y, Hu G, Lin HC, Hong SJ, Deng YH, Tang JY, Seto SW, Kwan YW, Waye MM, Wang YT and Lee SM: Radix Astragali extract promotes angiogenesis involving vascular endothelial growth factor receptor-related phosphatidylinositol 3-kinase/ Akt-dependent pathway in human endothelial cells. Phytother Res 23: 1205-1213, 2009
10. Westerfield, M: A Guide for the Laboratory Use of Zebrafish (Danio rerio). The Zebrafish Book, 5th edition. University of Oregon Press, Eugene, 2007.

11. Merchan JR, Chan B, Kale S, Schnipper LE and Sukhatme VP: In vitro and in vivo induction of antiangiogenic activity by plasminogen activators and captopril. J Natl Cancer Inst 95: 388-399, 2003.

12. Sato Y and Rifkin DB: Autocrine activities of basic fibroblast growth factor: regulation of endothelial cell movement, plasminogen activator synthesis, and DNA synthesis. J Cell Biol 107: 1199-1205, 1988.

13. Risau W: Mechanisms of angiogenesis. Nature 386: 671-674, 1997.

14. Isner JM: Arterial gene transfer of naked DNA for therapeutic angiogenesis: early clinical results. Adv Drug Deliv Rev 30: 185-197, 1998.

15. Xiong Y, Huo Y, Chen C, Zeng H, Lu X, Wei C, Ruan C, Zhang $\mathrm{X}, \mathrm{Hu} \mathrm{Z}$, Shibuya $\mathrm{M}$ and Luo J: Vascular endothelial growth factor (VEGF) receptor-2 tyrosine 1175 signaling controls VEGF-induced von Willebrand factor release from endothelial cells via phospholipase C-gamma 1- and protein kinase A-dependent pathways. J Biol Chem 284: 23217-23224, 2009.

16. Lam HW, Lin HC, Lao SC, Gao JL, Hong SJ, Leong CW, Yue PY, Kwan YW, Leung AY, Wang YT and Lee SM: The angiogenic effects of Angelica sinensis extract on HUVEC in vitro and zebrafish in vivo. J Cell Biochem 103: 195-211, 2008.

17. Hong SJ, Wan JB, Zhang Y, Hu G, Lin HC, Seto SW, Kwan YW, Lin ZX, Wang YT and Lee SM: Angiogenic effect of saponin extract from Panax notoginseng on HUVECs in vitro and zebrafish in vivo. Phytother Res 23: 677-686, 2009.

18. Sengupta S, Toh SA, Sellers LA, Skepper JN, Koolwijk P, Leung HW, Yeung HW, Wong RN, Sasisekharan R and Fan TP: Modulating angiogenesis: the yin and the yang in ginseng. Circulation 110: 1219-1225, 2004.

19. Tang JY, Li S, Li ZH, Zhang ZJ, Hu G, Cheang LC, Alex D, Hoi MP, Kwan YW, Chan SW, Leung GP and Lee SM: Calycosin promotes angiogenesis involving estrogen receptor and mitogen-activated protein kinase (MAPK) signaling pathway in zebrafish and HUVEC. PLoS One 5: e11822, 2010.

20. Tanimoto T, Jin ZG and Berk BC: Transactivation of vascular endothelial growth factor (VEGF) receptor Flk-1/KDR is involved in sphingosine 1-phosphate-stimulated phosphorylation of Akt and endothelial nitric-oxide synthase (eNOS). J Biol Chem 277: 42997-43001, 2002.

21. Ahmad S, Hewett PW, Wang P, Al-Ani B, Cudmore M, Fujisawa T, Haigh JJ, le Noble F, Wang L, Mukhopadhyay D and Ahmed A: Direct evidence for endothelial vascular endothelial growth factor receptor-1 function in nitric oxide-mediated angiogenesis. Circ Res 99: 715-722, 2006. 